\title{
Success and complications by team composition for prehospital paediatric intubation: a systematic review and meta-analysis
}

Alan A. Garner ${ }^{1,2^{*}}$, Nicholas Bennett ${ }^{2}$, Andrew Weatherall ${ }^{1,3}$ and Anna Lee L $^{4,5}$

\begin{abstract}
Background: Clinical team composition for prehospital paediatric intubation may affect success and complication rates. We performed a systematic review and meta-analysis to determine the success and complication rates by type of clinical team.

Methods: We searched MEDLINE, EMBASE, and CINAHL for interventional and observational studies describing prehospital intubation attempts in children with overall success, first-pass success, and complication rates. Eligible studies, data extraction, and assessment of risk of bias were assessed independently by two reviewers. We performed a random-effects meta-analysis of proportions.

Results: Forty studies (1989 to 2019) described three types of clinical teams: non-physician teams with no relaxants (22 studies, $n=7602$ ), non-physician teams with relaxants (12 studies, $n=2185$ ), and physician teams with relaxants (12 studies, $n=1780)$. Twenty-two $(n=3747)$ and $18(n=7820)$ studies were at low and moderate risk of bias, respectively. Non-physician teams without relaxants had lower overall intubation success rate $(72 \%, 95 \% \mathrm{Cl} 67-76 \%)$ than non-physician teams with relaxants (95\%, 95\% Cl 93-98\%) and physician teams (99\%, 95\% Cl 97-100\%). Physician teams had higher first-pass success rate (91\%, 95\% Cl 86-95\%) than non-physicians with $(75 \%$, 95\% Cl $69-$ $81 \%)$ and without $(55 \%, 95 \% \mathrm{Cl} 48-63 \%)$ relaxants. Overall airway complication rate was lower in physician teams (10\%, 95\% Cl 3-22\%) than non-physicians with (30\%, 95\% Cl 23-38\%) and without (39\%, 95\% Cl 28-51\%) relaxants.

Conclusion: Physician teams had higher rates of intubation success and lower rates of overall airway complications than other team types. Physician prehospital teams should be utilised wherever practicable for critically ill children requiring prehospital intubation.
\end{abstract}

Keywords: Airway management, Child, Complications, Emergency medical services, Intubation

\footnotetext{
* Correspondence: alan.garner@careflight.org

${ }^{1}$ CareFlight Australia, 4 Barden St, Northmead, NSW 2152, Australia

${ }^{2}$ The University of Sydney, Sydney, Australia

Full list of author information is available at the end of the article
}

(c) The Author(s). 2020 Open Access This article is licensed under a Creative Commons Attribution 4.0 International License, which permits use, sharing, adaptation, distribution and reproduction in any medium or format, as long as you give appropriate credit to the original author(s) and the source, provide a link to the Creative Commons licence, and indicate if changes were made. The images or other third party material in this article are included in the article's Creative Commons licence, unless indicated otherwise in a credit line to the material. If material is not included in the article's Creative Commons licence and your intended use is not permitted by statutory regulation or exceeds the permitted use, you will need to obtain permission directly from the copyright holder. To view a copy of this licence, visit http://creativecommons.org/licenses/by/4.0/ The Creative Commons Public Domain Dedication waiver (http://creativecommons.org/publicdomain/zero/1.0/) applies to the data made available in this article, unless otherwise stated in a credit line to the data. 


\section{Background}

Airway management is a critical component of prehospital care for severely ill and injured children. Airway management is arguably even more important in children than in adults, as cardiac arrest is more likely to be hypoxic in origin and therefore amenable to airway and ventilation intervention. As hypoxia correction is a time critical intervention, an emergency medical service (EMS) system must be able to provide airway management as early as possible, preferably at the incident scene.

Intubation is generally considered to be the gold standard for airway management in the critically ill and injured. Children however typically comprise only about $5 \%$ of total EMS cases [1-3], and those requiring intubation vary from $0.1 \%$ of all EMS responses $[3,4]$ to approximately $5 \%$ of paediatric cases when advanced intervention teams are selectively utilised [1, 2]. Success rates are also reported to be lower in children and the complication rate higher $[5,6]$. Traditionally, ground EMS systems have intubated children without muscle relaxants, but many systems are introducing relaxants into their clinical protocols with the expectation that overall success rates would improve and that intubation could be offered for a wider range of pathologies. There are also recent reports that physician staffed helicopter EMS (PS-HEMS) may produce particularly high procedural success with low complication rates [7-10].

The purpose of this study was to systematically review the available literature and perform a meta-analysis to determine whether there exists an association between type of prehospital team and intubation success and complication rates.

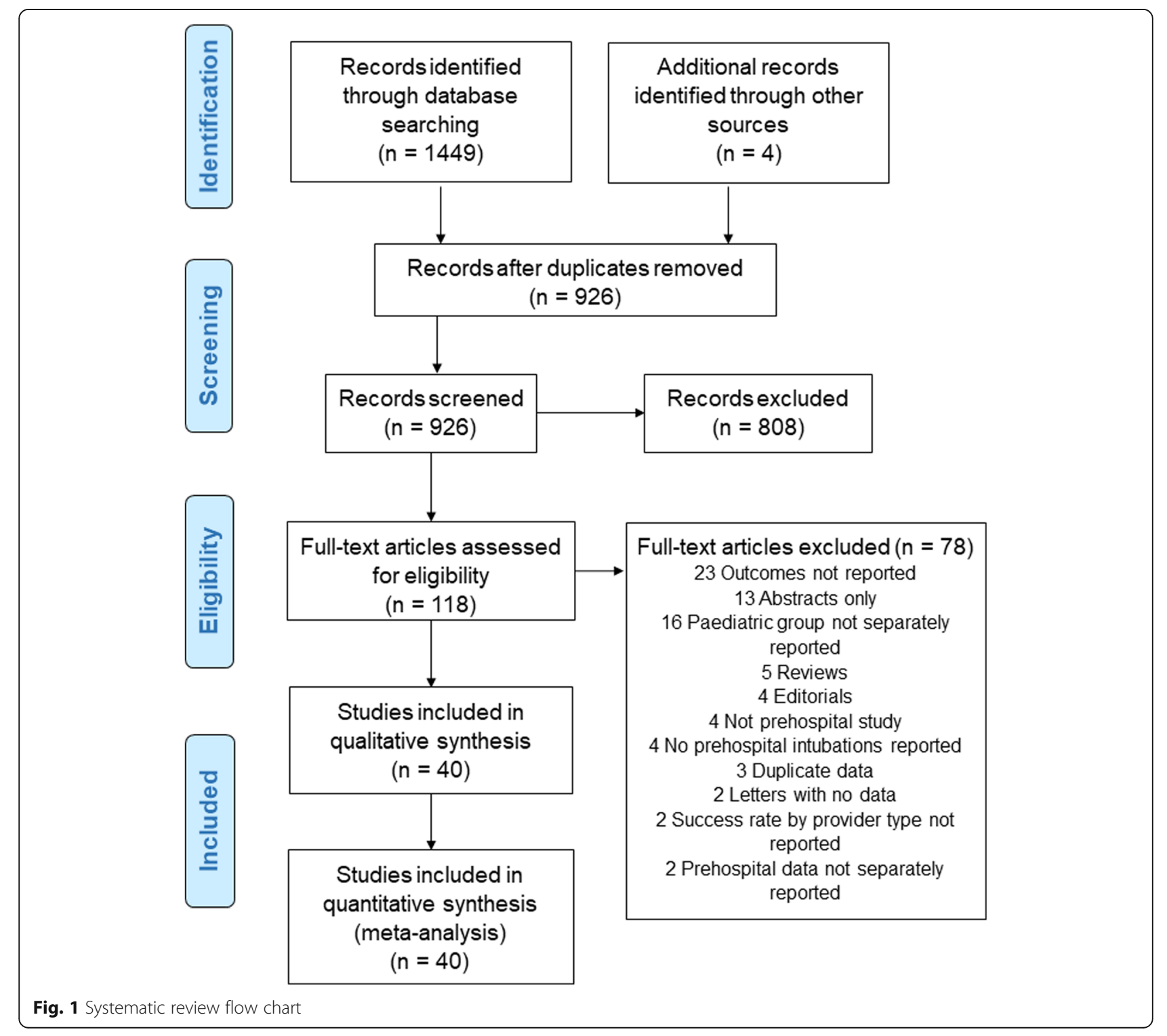




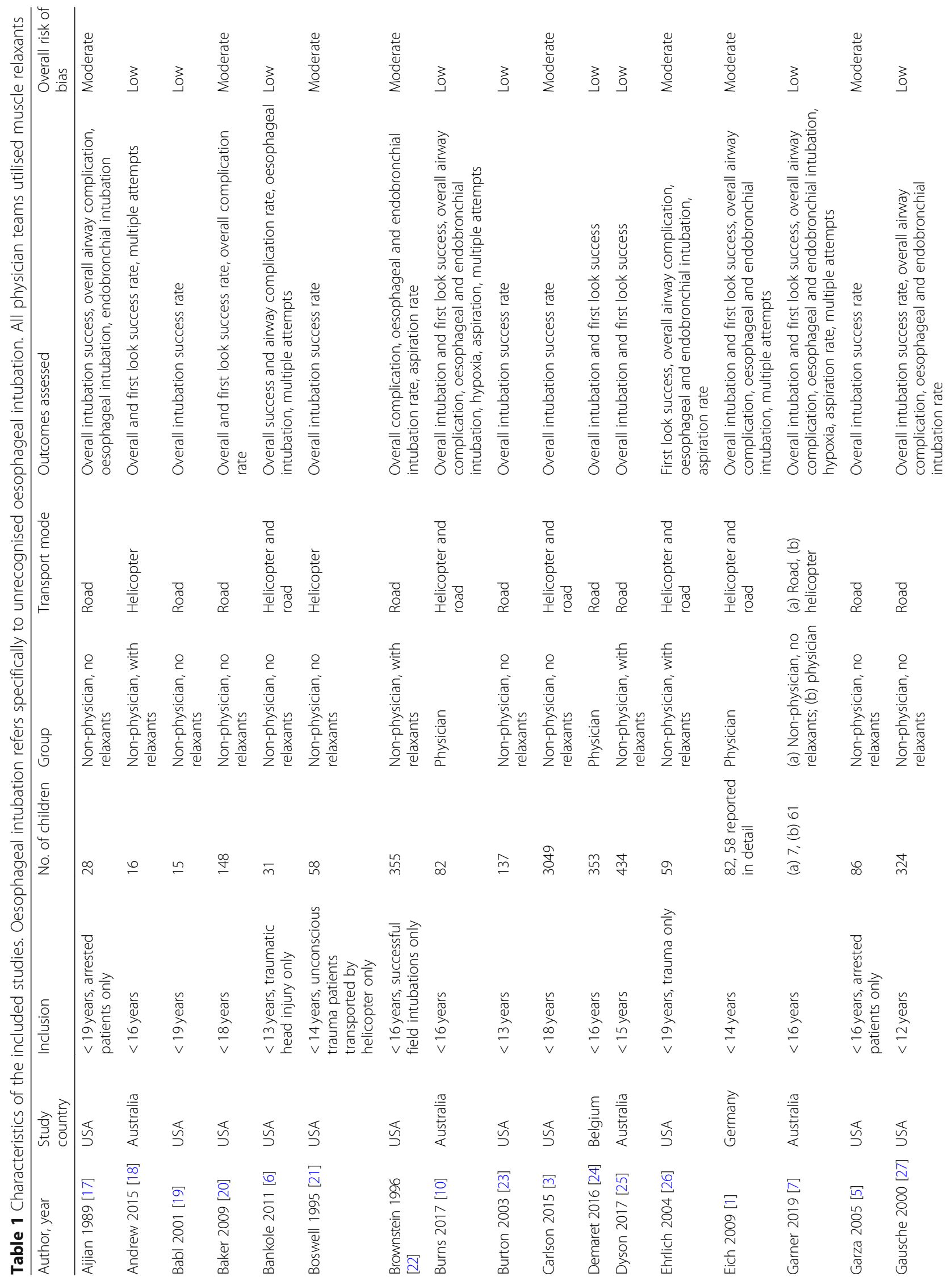




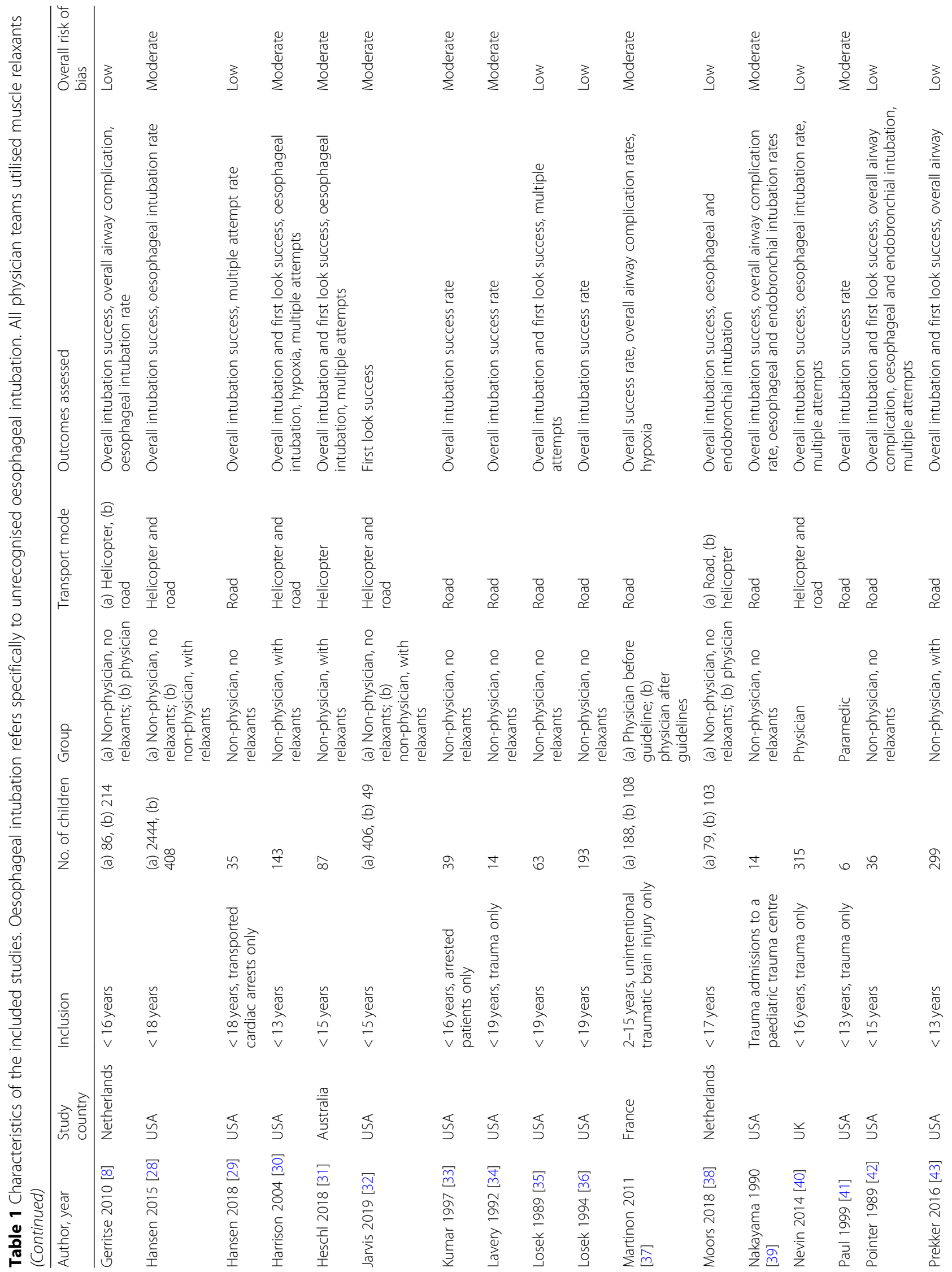




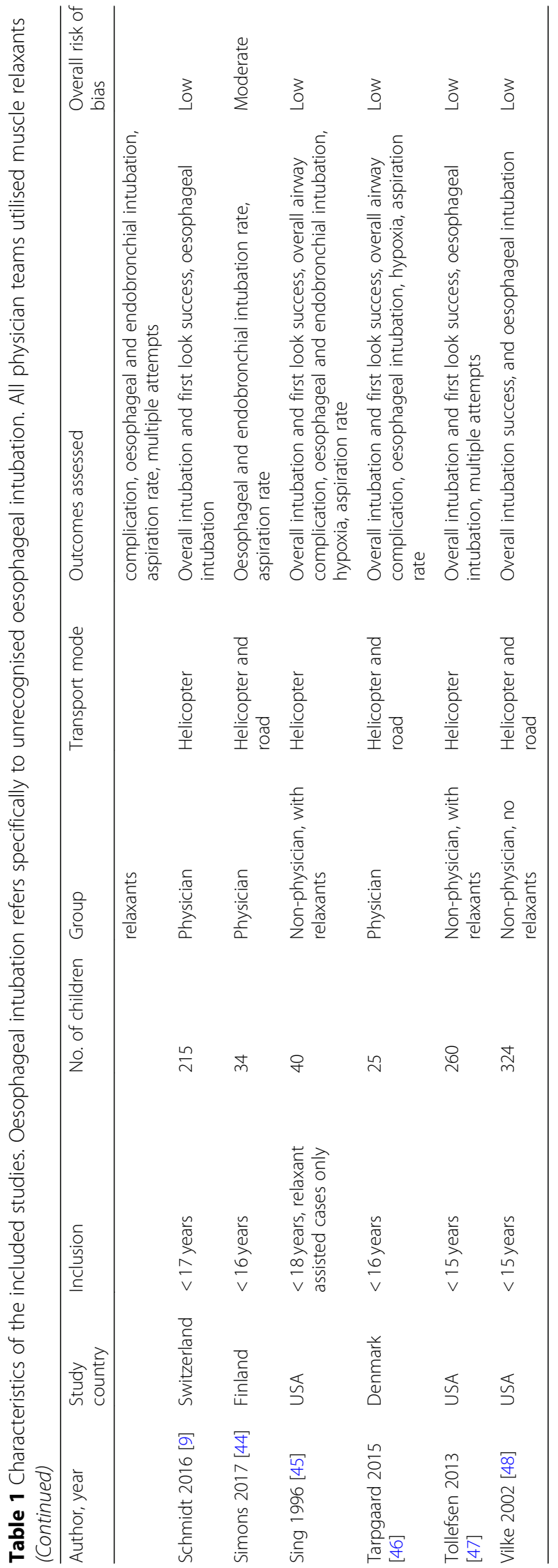


We hypothesised that utilisation of muscle relaxants by non-physician teams would improve procedural success in prehospital paediatric intubation over teams without relaxant access and that the greater experience and training of physician teams might produce further performance gains above those associated with relaxant access for non-physician teams.

\section{Methods}

The systematic review was conducted and reported in accordance with the PRISMA (Preferred Reporting Items for Systematic Reviews and Meta-Analyses) Guidelines [11].

\section{Data sources and literature search strategy}

We created search strategies for the concepts of 'intubation', 'prehospital', and 'paediatric' using a combination of standardised terms and keywords drawn from indices, thesauri, and on-topic articles (Supplementary eAppendix) in consultation with a medical librarian. The electronic databases Ovid MEDLINE, EMBASE, and CINAHL were searched from database inception to November 11, 2019. Additionally, we conducted a manual search of reference lists of included and other relevant articles. All articles were reviewed for inclusion by two independent reviewers (AG and NB). Any discrepancies were resolved by consensus with a third reviewer (AW).

\section{Study selection}

Interventional and/or observational studies were eligible for inclusion if they reported data on the success, firstpass success, and/or complication rates of prehospital paediatric intubation attempts. Studies that did not separately report the number of patients in whom intubation was attempted were excluded from analysis, as were abstract-only and grey literature reports. There was no language restriction.

\section{Data extraction}

Successful intubation, first-pass success rate, and complication rates were extracted from the included articles by two independent investigators (AG and $\mathrm{NB}$ ). Where there was discrepancy, a third author (AL) adjudicated. We also extracted data about authors, publication year, study location, setting, professional background of team members, availability of muscle relaxants, and participant characteristics by inclusion age and intubation indication. For each study, the team composition (exposure variable) was classified into three groups: non-physicians with no relaxants, non-physicians with relaxants, and physicians with relaxants. We made no contact with authors for missing data as many studies were old.

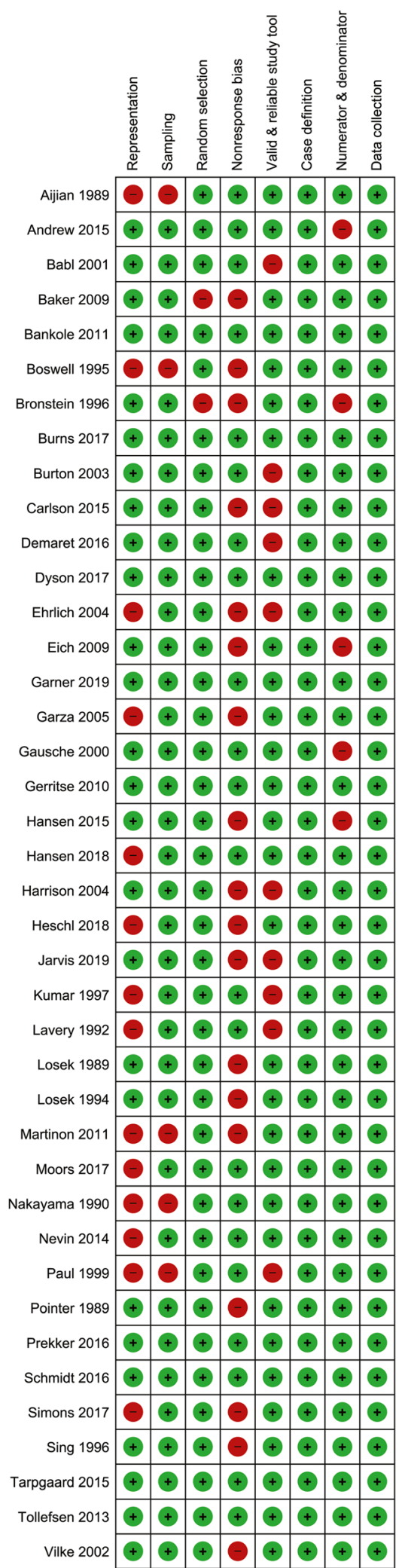

Fig. 2 Review authors' judgements about each risk of bias item for each included study. Low and high risk of bias are represented as green and red symbols, respectively 


\section{Outcomes}

The primary outcomes were the proportions of overall intubation and first look success rates. Secondary outcomes included the rate of intubation complications, specifically unrecognised oesophageal and endobronchial intubation, three or more attempts at intubation, hypoxia, or aspiration.

\section{Assessment of study quality}

The criteria used by Fouche and colleagues [12] were used to evaluate study quality. The checklist consists of 8 items that assess external and internal validity through 4 domains: selection bias, non-response bias, measurement bias, and bias related to the analysis, with each item graded as low or high [12]. The overall risk of bias for the study was rated 'low' if 7 or more domains were rated low, 'moderate' if 4 to 6 domains were rated low, and 'high' if 1 to 3 domains were rated low [12]. Each included study was assessed by AL and reviewed by AG.

\section{Data synthesis and statistical analysis}

We used the macro 'metaprop_one' in STATA 16.0 (StataCorp, College Station, TX) to pool proportions using the Freeman-Tukey double arcsine option to ensure that the confidence intervals around the estimates did not fall outside 0 or 1 with stable variances [13]. We used a logistic-normal random-effects model [13] and assessed the heterogeneity as low, moderate, and high using $I^{2}$ values of $25 \%, 50 \%$, and $75 \%$ [14]. We performed subgroup analyses by team composition a priori to explain heterogeneity and conducted a sensitivity analysis on low risk of bias trials to estimate the robustness of primary outcome results. Meta-regression with robust variance estimates (to take into account within-study correlation between different team types) was used to explore differences in the primary outcomes by team composition subgroups over time (year of publication) [15]. As there were large variations in the clinical population (mixed, trauma, head injury, and arrested) studied, subgroup meta-analyses by team composition were also performed for the overall intubation rate, first-pass success rate, and overall airway complication rate. We did not assess publication bias with a funnel plot as it has been shown to be problematic in meta-analysis of proportions [16].

\section{Results}

\section{Search results}

The search strategy yielded 40 eligible studies included in the analysis (Fig. 1). The characteristics of 40 included studies involving 11,567 children are shown in Table 1 [1, 3, 510, 17-48]. Fifteen studies (8201 participants) were published from 2015 onwards. The median (IQR) sample size of the studies was 86 (36 to 270). Twenty-six studies were published in the USA, five in Australia, two in the Netherlands, and one each in Belgium, Denmark, Finland, France, Germany, Switzerland, and the UK from 1989 to 2019.

Five studies [7, 8, 28, 32, 38] compared outcomes between different intubator groups, and one study [37] described outcomes before and after implementation of national guidelines. Of the 46 described team compositions, 22 studies utilised non-physicians with no relaxants $(n=7602), 12$ utilised non-physicians with relaxants $(n=2185)$, and 12 utilised physicians, all with relaxants $(n=1780)$. Studies published before 2010 were mainly non-physicians with no relaxants $(15 / 20)$. Since 2010, 11 of 12 studies involved physicians. Mode of transportation was road (24/46), helicopter (10/46), and both road and helicopter (12/46).

\section{Quality assessment}

None of the 40 studies were rated as high risk of bias. Eighteen studies $(n=7820)$ were rated moderate risk of

A close representation of national population

True or close representation of target population

Random selection or census

Nonresponse bias minimal

Valid and reliable study tool

Acceptable case definition

Numerator and denominator appropriate

Same mode of data collection

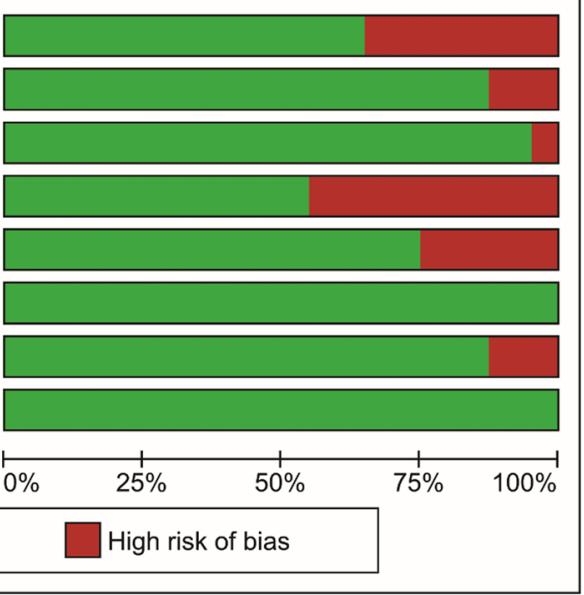

Fig. 3 Review authors' judgements about each risk of bias item presented as percentages across all included studies 
bias, and 22 studies $(n=3747)$ had overall low risk of bias. Main reasons for biases for individual studies are shown in Fig. 2. Selection bias (items 1 to 3) was present in 16 studies (Fig. 3). Non-response bias (item 4) more than $20 \%$ was the most common type of bias affecting external validity (Fig. 3).

\section{Overall intubation and first-pass success rates}

Thirty-five studies (40 reports by a specific team type) in 10,456 children examined the overall success intubation rates, with most data coming from nonphysician teams without relaxants $(n=7181)$. There was a significant intra-group heterogeneity $\left(I^{2}>72 \%\right.$

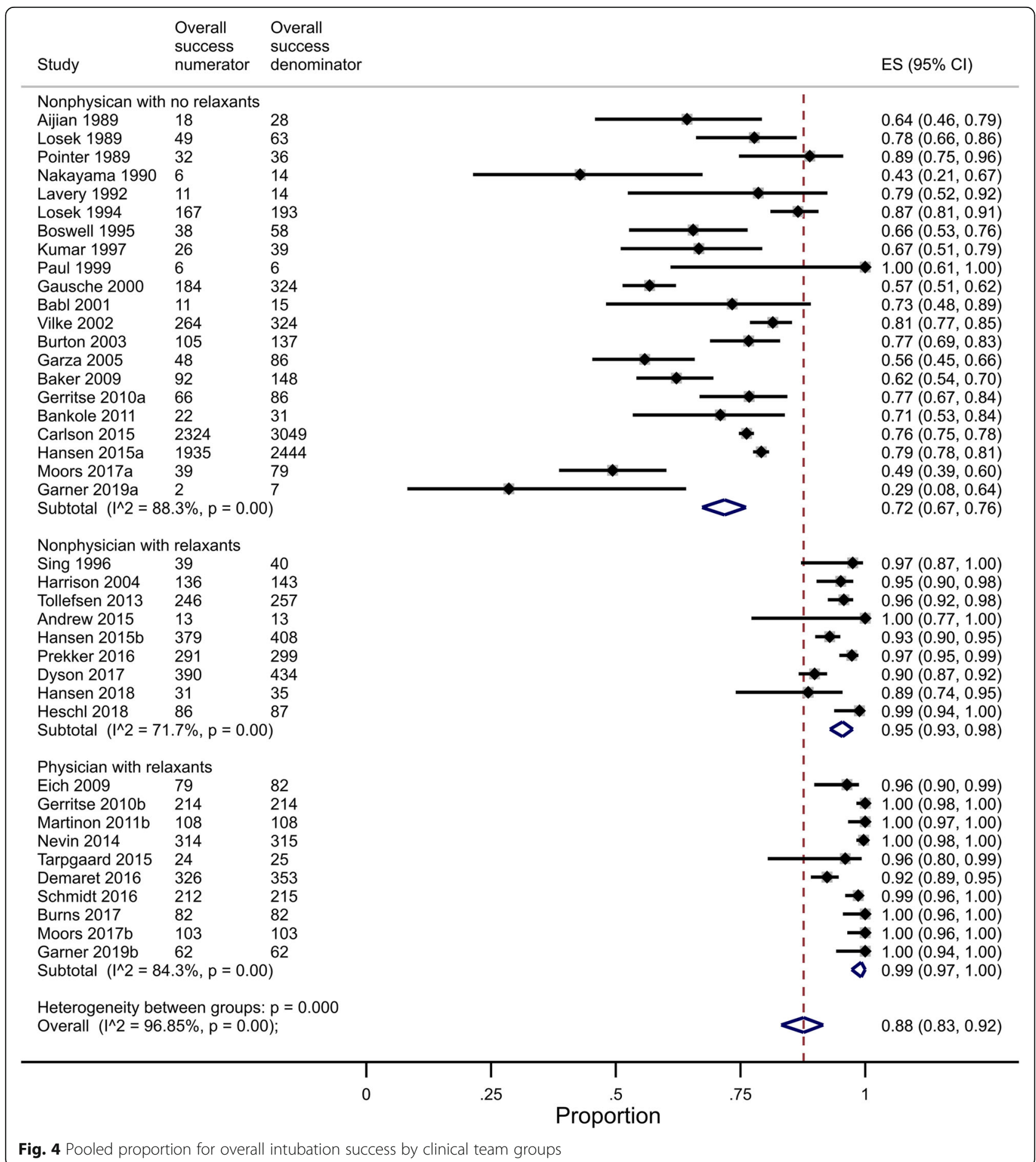


for all three team composition groups) and a significant inter-group heterogeneity $(P<0.001)$, supporting the separate reporting of overall success intubation rates by subgroups. Non-physician teams without relaxants had lower overall success rates $(72 \%, 95 \%$ CI 67-76\%) than non-physician teams with relaxants (95\%, 95\% CI 93-98\%) and physician teams (99\%, 95\% CI 97-100\%) (Fig. 4). Differences in overall success rates by team composition were significant $(P<$ $0.001)$ after adjusting for time $(-0.3 \% /$ year, $95 \% \mathrm{CI}-$ $0.8 \% /$ year to $0.3 \% /$ year, $P=0.29$ ) (Fig. 5 ).

The sensitivity analysis of 21 low risk of bias studies (24 team composition groups) in 3707 children showed similar results, with non-physician teams without relaxants having lower overall success rates (73\%, 95\% CI 64-81\%) than non-physician teams with relaxants (96\%, 95\% CI 92-99\%) and physician teams (99\%, 95\% CI 97-100\%). Meta-regression in low risk of bias studies showed that team composition differences in overall success rates remained significant $(P<0.001)$ after adjusting for time $(-0.9 \% /$ year, $95 \%$ CI $-0.1 \% /$ year to $-1.6 \%$ /year, $P=0.04$ ).

Eighteen studies in 2752 children examined firstpass success rates (Fig. 6). There was a significant intra-group heterogeneity $(P<0.001)$ and a significant inter-group heterogeneity $(P<0.001)$, supporting the separate reporting of first-pass success intubation rates by subgroups. Physician teams had significantly higher first-pass success rates $(91 \%, 95 \%$ CI $86-95 \%)$ than non-physician teams with relaxants $(75 \%, 95 \%$ CI $69-81 \%)$ or without relaxants $(55 \%, 95 \%$ CI $48-$ $63 \%)$. Meta-regression was problematic as the degrees of freedom were less than 4 [15]. Sensitivity analysis in 12 low risk of bias studies $(n=1843)$ showed significant inter-group heterogeneity $(P<0.001)$, with physician teams associated with higher first-pass

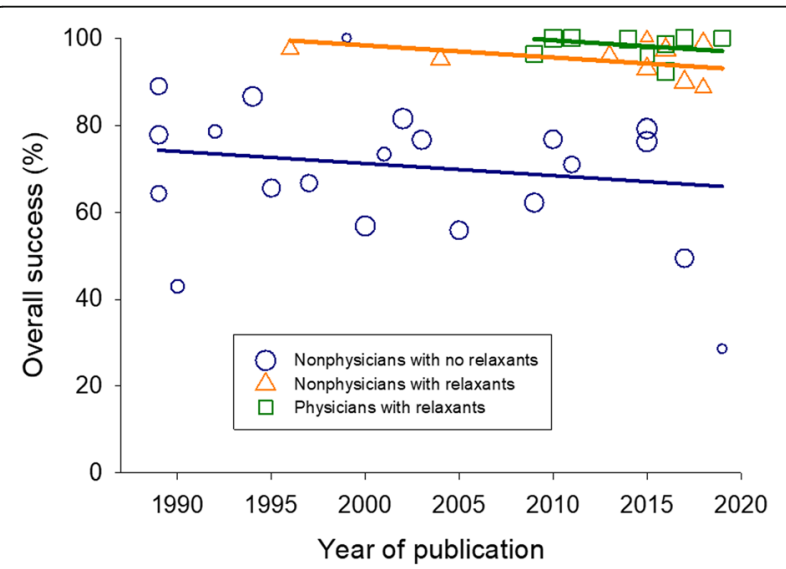

Fig. 5 Meta-regression of overall intubation success over time (year of publication). Meta-regression lines are drawn for each clinical team group success rates (92\%, 95\% CI 87-96\%) than nonphysician teams with relaxants $(73 \%, 95 \%$ CI $67-78 \%)$ or without relaxants (47\%, 95\% CI 35-59\%).

\section{Adverse events}

Most adverse events by team type showed large intragroup and inter-group heterogeneity, supporting the need to report individual team pooled estimates (Table 2). Sixteen studies, involving 1975 children, examined the overall intubation complication rate. The overall airway complication rate was lower in physician teams than in non-physicians with and without relaxants (Table 2). Physician teams were not associated with the occurrence of oesophageal intubations, aspirations, or the need for three or more multiple intubation attempts $(P=1.00, \quad P=0.27, \quad P=0.24$, respectively).

As there was no inter-group heterogeneity for endobronchial intubation $(P=0.15)$, the overall pooled estimated was $7 \%$ (95\% CI 3-12\%) (Table 2). However, Simons and colleagues' study [44] appears to be an outlier $(21 \%, 95 \%$ CI $10-37 \%)$ as there were exhaustive attempts to determine the endotracheal tube position after arrival in the emergency department. A post hoc sensitivity analysis, excluding Simons and colleagues' study [44], showed that there was a significant inter-group heterogeneity $(P<0.001)$, with a pooled estimate for physician team decreasing to $0 \%$ (95\% CI 0-2\%).

Of the 40 studies included in this systematic review, only six [7, 10, 30, 37, 45, 46] (651 children) reported hypoxia after intubation. Much of the meta-analysis result was influenced by Martinon and colleagues' study [37] $(n=296)$ that examined the effect of national guidelines on prehospital intubation in severely head-injured children. A post hoc sensitivity analysis, excluding Martinon and colleagues' study [37], showed that there was a significant inter-group heterogeneity $(P<0.001)$, with a pooled estimate for physician team decreasing to $3 \%(95 \%$ CI $0-10 \%)$.

\section{Outcomes by clinical population}

Seven studies were in trauma patients $(n=500)$ [21, $26,34,39-41,44]$, six in arrested patients $(n=804)$ $[5,17,25,29,33,38]$, and three in head injury patients $(n=414)[6,31,37]$, and the remaining studies comprised a mixed population of all patients requiring airway management $(n=9849)$. There was no association between team composition and type of paediatric patients treated $(P=0.875)$. Non-physicians with or without relaxants had lower rates of overall intubation success in arrested patients compared with other patient populations (Table 3). The first-pass success rate and 


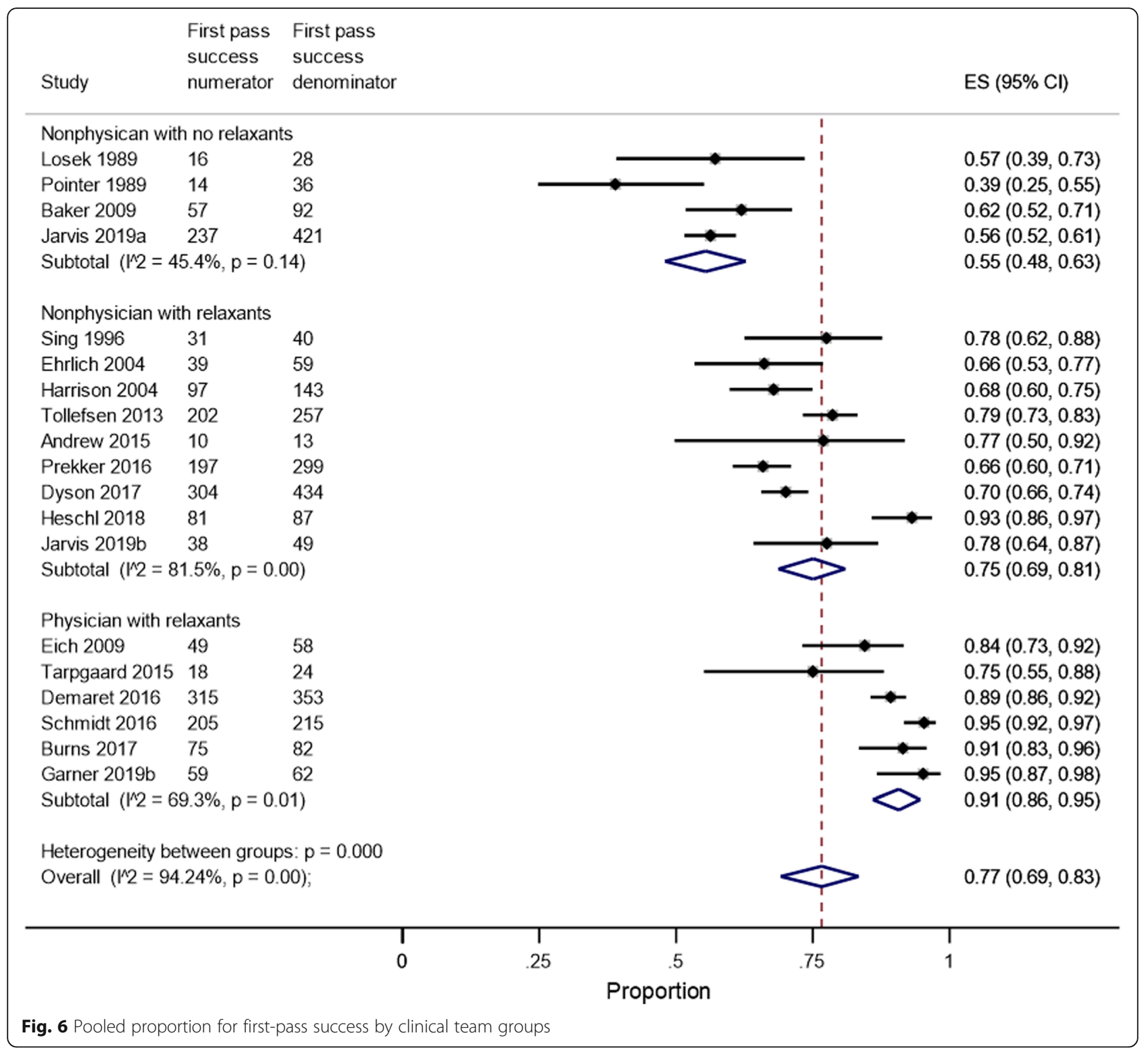

overall airway complication rate by patient population and team composition are shown in Table 3.

\section{Discussion}

To our knowledge, this is the first meta-analysis to compare prehospital intubation success and complication rates of different teams of intubator providers specifically in children. The success and complication rates for physician teams are better than nonphysician teams either with or without muscle relaxants. Although reported clinical populations varied between studies, the success and complications rates followed the same pattern when population subgroup meta-analyses were performed. The overall success and first-pass success estimates were robust in the sensitivity analyses. Even after adjusting for the year of publication in the meta-regressions, team composition differences in the overall success estimate remained significant. The overall quality of evidence was graded as moderate to high after assessing for the presence of selection and non-response bias, measurement bias, and bias related to data analysis [12].

Two previous meta-analyses [12, 49] examining the success and complication rates by physician and nonphysician teams regardless of patient age where both team types utilised relaxants demonstrated higher overall and first-pass success for physician teams compared with non-physician teams. Our review indicates that this is also observed in the paediatric subgroup. A possible contributor to higher success rates by physician teams is in-hospital exposure to 


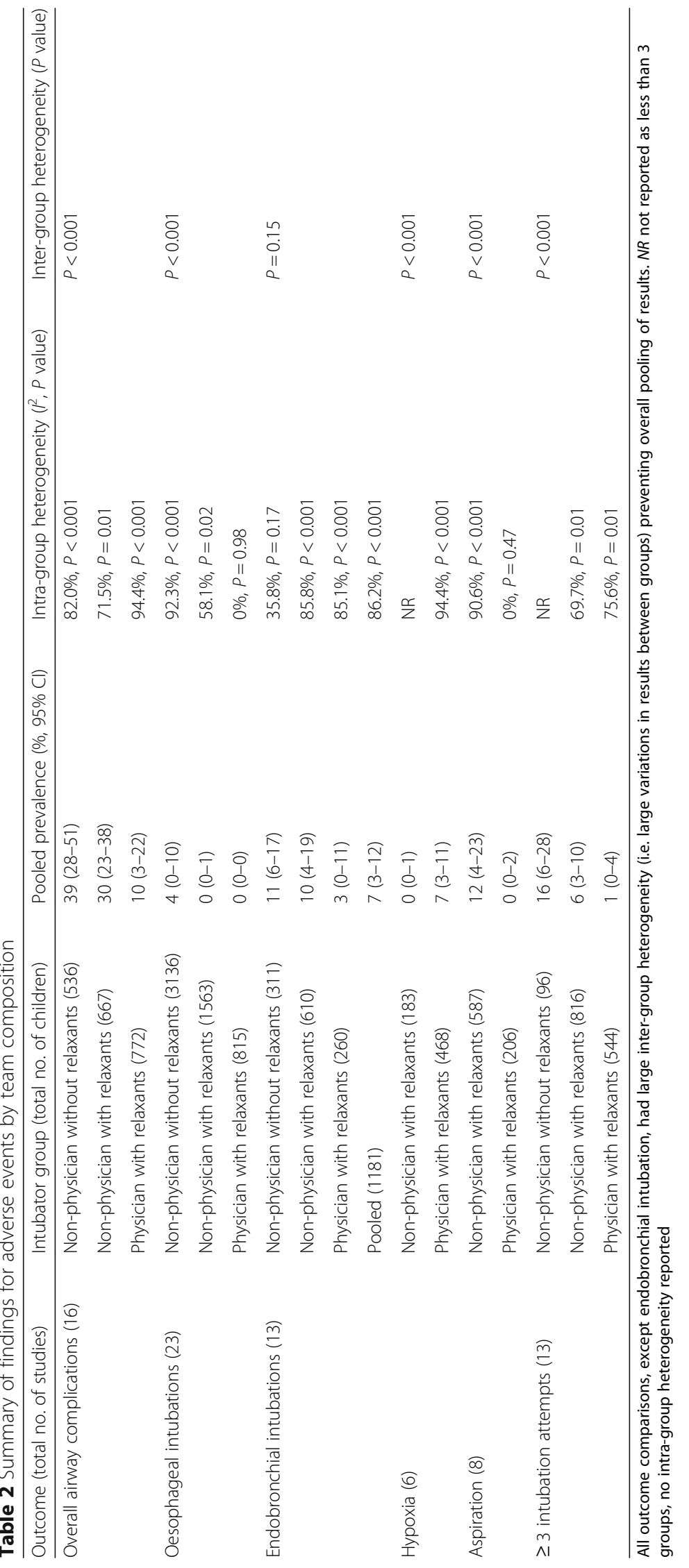




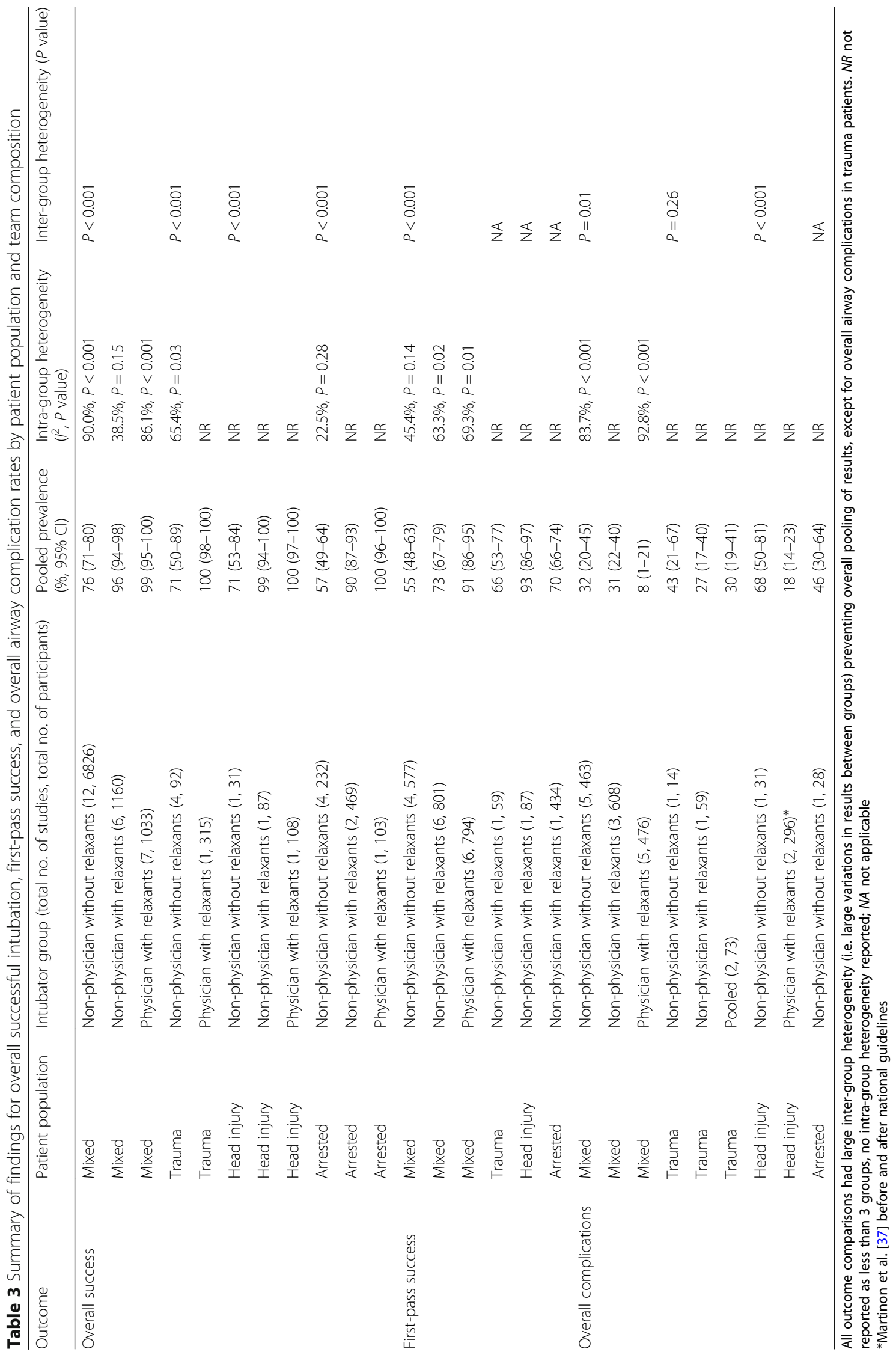


paediatric intubation compensating for the rare requirement for this procedure in prehospital practice. All of the identified physician team studies utilised HEMS for at least some responses, and it may be that there is an additive effect from HEMS increasing team experience by allowing small numbers of clinicians to cover a larger population thereby concentrating exposure. As non-physician teams utilising relaxants have higher success rates when transported by HEMS compared with ground transport lends additional support to this theory.

Successful use of a clinical bundle to avoid periintubation hypoxia by a non-physician team utilising relaxants in non-arrested adults has been reported [50]. The bundle mandated intubation attempts be abandoned in favour of mask ventilation and urgent transport when pre-oxygenation failed to achieve a $\mathrm{SpO}_{2}$ of at least $94 \%$. The complete bundle reduced peri-intubation hypoxia rates from 44.2 to $3.5 \%$ and suggests that avoiding prehospital intubation in hypoxic patients may minimise risk for teams with lower experience levels. This approach however also potentially denies intubation to patients with critical hypoxia who are arguably the most likely to benefit from early intubation. A focus on oxygenation rather than procedural success is suggested for future studies given this is the primary aim of all airway management. It is noteworthy that in our systematic review, only six studies could be identified that reported hypoxia as an outcome from 40 studies that met the inclusion criteria.

Caution is also needed in interpreting our metaanalysis subgroup analysis results as these are observational in nature. However, we believe that the results of the within-study comparisons of different team composition performances in four studies $[8,28$, $32,38]$, together with insights from our recent study [7], are credible and supportive of higher overall intubation success, first-pass success, and lower complication rates associated with physician teams. Our results were also robust when sensitivity analysis and meta-regressions were performed. The definition of paediatric age group varied between studies ranging from $<13$ to $<19$ years. Inclusion of a large proportion of teenage patients in a sample is unlikely to reflect the specific issues of paediatric airway management as the greatest difficulty and complication rates occur in smaller children.

Differences in airway training between studies and between team types are a possible explanation for the observed performance differences. As a major difference between physicians and non-physicians is the training programmes to which they have been exposed, it is intuitive to suggest that further training of non-physician teams may decrease or eliminate the observed differences. Reporting of airway training was too heterogeneous to support an analysis however. Some studies provided no description of training [19, $21,24,26,39,44,45]$, and some reported pooled data from multiple agencies $[3,28,32]$, whilst others described the studied teams simply as Advanced Life Support and/or Paediatric Advanced Life Support certified [5, 20, 33-35].

It is possible that there is variability between team types in willingness to report complications. Studies have demonstrated under-reporting of prehospital intubation complications by non-physician personnel [51] and physician teams [7] when documentation is compared with electronic monitor data. Similarly, under-reporting has been documented in the emergency department setting when video recordings of the resuscitation are reviewed [52]. We are not aware of any studies that compare the rates of underreporting between team types however. Underreporting is also likely to be affected by factors such as organisational and national cultures which may confound any difference by team type as well as the status of legal protection for disclosure of complications in the reporting jurisdiction. Ideally, future studies should report complications based on monitor data and/or video review.

\section{Conclusions}

Our systematic review supports higher overall success and first-pass success with lower complication rates by teams incorporating physicians when intubating children in the prehospital environment. The results of the metaanalysis suggest that this applies regardless of nonphysician team utilisation of neuromuscular blockade. Physician prehospital teams should be utilised wherever practicable for critically ill children requiring prehospital intubation.

\section{Supplementary information}

Supplementary information accompanies this paper at https://doi.org/10. 1186/s13054-020-02865-y.

Additional file 1.

Acknowledgements

We thank the staff at Nepean Hospital, Sydney, for their help with the electronic database searches for the systematic review.

\section{Authors' contributions}

Conceived the study: A.A.G., A.W., A.L. Designed the protocol: A.A.G., A.W., A.L. Collected the data: A.A.G., N.B., A.W., A.L. Analysed the data: A.A.G., A.L., with input from A. W, N.B. Drafted the manuscript: A.A.G., A.L., revised following critical review by all authors. The authors read and approved the final manuscript. 


\section{Funding}

This study was supported by internal funds from CareFlight and the Chinese University of Hong Kong. The funders had no role in the design and conduct of the study, collection, management, analysis, and interpretation of the data; preparation, review, or approval of the manuscript; and decision to submit the manuscript for publication.

\section{Availability of data and materials}

The datasets used and/or analysed during the current study are available from the corresponding author on reasonable request.

\section{Ethics approval and consent to participate}

Not applicable to a systematic review.

\section{Consent for publication}

Not applicable to a systematic review.

\section{Competing interests}

A.L. is an editor for the Cochrane Anaesthesia and the Cochrane Emergency and Critical Care Review Groups and is a member of the editorial board for Perioperative Medicine Journal. All other authors declare no conflicts of interest.

\begin{abstract}
Author details
${ }^{1}$ CareFlight Australia, 4 Barden St, Northmead, NSW 2152, Australia. ${ }^{2}$ The University of Sydney, Sydney, Australia. ${ }^{3}$ Division of Paediatrics and Child Health, The University of Sydney, Sydney, Australia. ${ }^{4}$ Department of Anaesthesia and Intensive Care, The Chinese University of Hong Kong, Shatin, Hong Kong. ${ }^{5}$ Hong Kong Branch of The Chinese Cochrane Centre, The Jockey Club School of Public Health and Primary Care, Faculty of Medicine, The Chinese University of Hong Kong, Shatin, Hong Kong.
\end{abstract}

\section{Received: 1 January 2020 Accepted: 31 March 2020}

\section{Published online: 15 April 2020}

\section{References}

1. Eich C, Roessler M, Nemeth M, Russo SG, Heuer JF, Timmermann A. Characteristics and outcome of prehospital paediatric tracheal intubation attended by anaesthesia-trained emergency physicians. Resuscitation. 2009; 80:1371-7.

2. Nagele P, Kroesen G. Pediatric emergencies. An epidemiologic study of mobile care units in Innsbruck. Anaesthesist. 2000;49:725-31.

3. Carlson JN, Gannon E, Mann N, et al. Pediatric out-of-hospital critical procedures in the United States. Pediatr Crit Care Med. 2015;16:e260-7.

4. Richard J, Osmond MH, Nesbitt L, Stiell IG. Management and outcomes of pediatric patients transported by emergency medical services in a Canadian prehospital system. Can J Emerg Med. 2006;8:6-12.

5. Garza AG, Algren DA, Gratton MC, Ma OJ. Populations at risk for intubation nonattempt and failure in the prehospital setting. Prehosp Emerg Care. 2005;9:163-6.

6. Bankole $\mathrm{S}$, Asuncion $\mathrm{A}$, Ross $\mathrm{S}$, et al. First responder performance in pediatric trauma: a comparison with an adult cohort. Pediatr Crit Care Med. 2011;12:e166-70.

7. Garner AA, Bennett N, Weatherall A, Lee A. Physician-staffed helicopter emergency medical services augment ground ambulance paediatric airway management in urban areas: a retrospective cohort study. Emerg Med J. 2019;36:678-83.

8. Gerritse BM, Schalkwijk A, Pelzer BJ, Scheffer GJ, Draaisma JM. Advanced medical life support procedures in vitally compromised children by a helicopter emergency medical service. BMC Emerg Med. 2010;10:6.

9. Schmidt AR, Ulrich L, Seifert B, Albrecht R, Spahn DR, Stein P. Ease and difficulty of pre-hospital airway management in 425 paediatric patients treated by a helicopter emergency medical service: a retrospective analysis. Scand J Trauma Resusc Emerg Med. 2016;24:22.

10. Burns BJ, Watterson JB, Ware S, Regan L, Reid C. Analysis of out-of-hospital pediatric intubation by an Australian helicopter emergency medical service. Ann Emerg Med. 2017;70:773-82.

11. Liberati A, Altman DG, Tetzlaff J, et al. The PRISMA statement for reporting systematic reviews and meta-analyses of studies that evaluate healthcare interventions: explanation and elaboration. BMJ. 2009;339:b2700.
12. Fouche PF, Stein C, Simpson P, Carlson JN, Doi SA. Nonphysician out-ofhospital rapid sequence intubation success and adverse events: a systematic review and meta-analysis. Ann Emerg Med. 2017;70:449-59.

13. Nyaga VN, Arbyn M, Aerts M. Metaprop: a Stata command to perform metaanalysis of binomial data. Arch Public Health. 2014;72:39.

14. Higgins JP, Thompson SG, Deeks JJ, Altman DG. Measuring inconsistency in meta-analyses. BMJ. 2003;327:557-60.

15. Tanner-Smith EE, Tipton E. Robust variance estimation with dependent effect sizes: practical considerations including a software tutorial in Stata and SPSS. Res Synth Methods. 2014;5:13-30.

16. Hunter JP, Saratzis A, Sutton AJ, Boucher RH, Sayers RD, Bown MJ. In metaanalyses of proportion studies, funnel plots were found to be an inaccurate method of assessing publication bias. J Clin Epidemiol. 2014;67:897-903.

17. Aijian $P$, Tsai A, Knopp R, et al. Endotracheal intubation of pediatric patients by paramedics. Ann Emerg Med. 1989;18:489-94.

18. Andrew E, de Wit A, Meadley B, Cox S, Bernard S, Smith K. Characteristics of patients transported by a paramedic-staffed helicopter emergency medical service in Victoria, Australia. Prehosp Emerg Care. 2015;19:416-24.

19. Babl F, Vinci R, Bauchner H, Mottley L. Pediatric pre-hospital advanced life support care in an urban setting. Pediatr Emerg Care. 2001;17:5-9.

20. Baker T, King W, Soto W, Asher C, Stolfi A, Rowin M. The efficacy of pediatric advanced life support training in emergency medical service providers. Pediatr Emerg Care. 2009;25:508-12.

21. Boswell W, McElveen N, Sharp M, Boyd C, Frantz E. Analysis of prehospital pediatric and adult intubation. Air Med J. 1995;14:125-8.

22. Brownstein D, Shugerman R, Cummings P, Rivara F, Copass M. Prehospital endotracheal intubation of children by paramedics. Ann Emerg Med. 1996; 28:34-9.

23. Burton J, Baumann M, Maoz T, Bradshaw J, Lebrun J. Endotracheal intubation in a rural EMS state: procedure utilisation and impact of skills maintenance guidelines. Prehosp Emerg Care. 2003;7:352-6.

24. Demaret $P$, Lebrun $F$, Devos $P$, et al. Pediatric pre-hospital emergencies in Belgium: a 2-year national descriptive study. Eur J Pediatr. 2016;175:921-30.

25. Dyson K, Bray J, Smith K, et al. Paramedic intubation experience is associated with successful tube placement but not cardiac arrest survival. Ann Emerg Med. 2017;70:382-90,

26. Ehrlich P, Seidman P, Atallah O, Haque A, Helmkamp J. Endotracheal intubations in rural pediatric trauma patients. J Pediatr Surg. 2004:39: 1376-80.

27. Gausche M, Lewis RJ, Stratton SJ, et al. Effect of out-of-hospital pediatric endotracheal intubation on survival and neurological outcome: a controlled clinical trial. JAMA. 2000;283:783-90.

28. Hansen M, Lambert W, Guise J, Warden C, Mann N, Wang H. Out-of-hospital pediatric airway management in the United States. Resuscitation. 2015;90:104-10.

29. Hansen M, Eriksson C, Skarica B, Meckler G, Guise JM. Safety events in pediatric out-of-hospital cardiac arrest. Am J Emerg Med. 2018;36:380-3.

30. Harrison $T$, Thomas $S$, Wedel $S$. Success rates of pediatric intubation by a non-physician-staffed critical care transport service. Pediatr Emerg Care. 2004;20:101-7.

31. Heschl S, Meadley B, Andrew E, Butt W, Bernard S, Smith K. Efficacy of prehospital rapid sequence intubation in paediatric traumatic brain injury: a 9 year observational study. Injury. 2018;49:916-20.

32. Jarvis $J L$, Wampler $D$, Wang HE. Association of patient age with first pass success in out-of-hospital advanced airway management. Resuscitation. 2019:141:136-43.

33. Kumar V, Bachman D, Kiskaddon R. Children and adults in cardiopulmonary arrest: are advanced life support guidelines followed in the prehospital setting? Ann Emerg Med. 1997;29:743-7.

34. Lavery R, Tortella B, Griffin C. The prehospital treatment of pediatric trauma. Pediatr Emerg Care. 1992;8:9-12.

35. Losek JD, Bonadio WA, Walsh-Kelly C, Hennes H, Smith DS, Glaeser PW. Prehospital pediatric endotracheal intubation performance review. Pediatr Emerg Care. 1989;5:1-4.

36. Losek JD, Szewczuga D, Glaeser PW. Improved prehospital pediatric ALS care after an EMT-paramedic clinical training course. Am J Emerg Med. 1994;12:429-32.

37. Martinon C, Duracher C, Blanot S, et al. Emergency tracheal intubation of severely head-injured children: changing daily practice after implementation of national guidelines. Pediatr Crit Care Med. 2011;12:65-70.

38. Moors XRJ, Rijs K, Den Hartog D, Stolker RJ. Pediatric out-of-hospital cardiopulmonary resuscitation by helicopter emergency medical service, 
does it has added value compared to regular emergency medical service? Eur J Trauma Emerg Surg. 2018:44:407-10.

39. Nakayama D, Gardner M, Rowe M. Emergency endotracheal intubation in pediatric trauma. Ann Surg. 1990;211:218-33.

40. Nevin D, Green S, Weaver A, Lockey D. An observational study of paediatric pre-hospital intubation and anaesthesia in 1933 children attended by a physician-led, pre-hospital trauma service. Resuscitation. 2014;85:189-95.

41. Paul T, Marias M, Pons P, Pons K, Moore E. Adult versus pediatric prehospital trauma care: is there a difference? J Trauma. 1999;47:455-9.

42. Pointer JE. Clinical characteristics of paramedics' performance of pediatric endotracheal intubation. Am J Emerg Med. 1989;7:364-6.

43. Prekker M, Delgado F, Shin J, et al. Pediatric intubation by paramedics in a large emergency medical services system: process, challenges, and outcomes. Ann Emerg Med. 2016;67:20-9.

44. Simons T, Söderlund T, Handolin L. Radiological evaluation of tube depth and complications of prehospital endotracheal intubation in pediatric trauma: a descriptive study. Eur J Trauma Emerg Surg. 2017:43:797-804.

45. Sing R, Reilly P, Rotondo M, Lynch M, McCans J, Schwab C. Out-of-hospital rapid-sequence induction for intubation of the pediatric patient. Acad Emerg Med. 1996;3:41-5.

46. Tarpgaard M, Hansen TM, Rognås L. Anaesthetist-provided pre-hospital advanced airway management in children: a descriptive study. Scand J Trauma Resusc Emerg Med. 2015;23:61.

47. Tollefsen WW, Brown CA, Cox KL, Walls RM. Two hundred sixty pediatric emergency airway encounters by air transport personnel: a report of the air transport emergency airway management (NEAR VI: "A-TEAM") project. Pediatr Emerg Care. 2013;29:963-8.

48. Vilke G, Steen P, Smith A, Chan T. Out-of-hospital pediatric intubation by paramedics: the San Diego experience. J Emerg Med. 2002;22:71-4.

49. Crewdson K, Lockey DJ, Røislien J, Lossius HM, Rehn M. The success of prehospital tracheal intubation by different pre-hospital providers: a systematic literature review and meta-analysis. Crit Care. 2017;21:31.

50. Jarvis JL, Gonzales J, Johns D, Sager L. Implementation of a clinical bundle to reduce out-of-hospital peri-intubation hypoxia. Ann Emerg Med. 2018;72: 272-9.

51. Walker RG, White $L$, Whitmore GN, et al. Evaluation of physiologic alterations during prehospital paramedic-performed rapid sequence intubation. Prehosp Emerg Care. 2018;22:300-11.

52. Kerrey BT, Rinderknecht AS, Geis GL, et al. Rapid sequence intubation for pediatric emergency patients: higher frequency of failed attempts and adverse effects found by video review. Ann Emerg Med. 2012;60:251-9.

\section{Publisher's Note}

Springer Nature remains neutral with regard to jurisdictional claims in published maps and institutional affiliations.

Ready to submit your research? Choose BMC and benefit from:

- fast, convenient online submission

- thorough peer review by experienced researchers in your field

- rapid publication on acceptance

- support for research data, including large and complex data types

- gold Open Access which fosters wider collaboration and increased citations

- maximum visibility for your research: over $100 \mathrm{M}$ website views per year

At $\mathrm{BMC}$, research is always in progress.

Learn more biomedcentral.com/submissions 